\title{
Recycled dishes form telescope network
}

\section{Africa refits redundant satellite dishes for radio astronomy.}

\section{BY LINDA NORDLING}

A geing satellite dishes, once the backbone of Africa's telecommunications system, are being given a new lease of life as radio telescopes.

The thrifty project aims to boost the skills of the continent's scientists as Africa prepares to host the US $\$ 2.1$ billion Square Kilometre Array (SKA), set to be the world's most powerful radio telescope when it is completed in the mid-2020s. In May, the SKA project leaders decided that Africa, with South Africa at the helm, would split the array with Australia and New Zealand (see Nature 485, 555-556; 2012). The SKA will detect radiation from the early Universe, giving clues to how the first stars and galaxies formed.

South Africa has a nucleus of radio-astronomy expertise, but the rest of the continent has few researchers with the skills to work on the SKA. The African SKA partners hope to train a new generation of radio astronomers using the converted satellite dishes, which have been superseded by the high-speed fibre-optic cables skirting Africa’s coast. "We want to make very sure that Africa delivers the best possible instrument, and that African engineers and scientists can maintain and support it," says Anita Loots, associate director of SKA South Africa.

The first dish, in Kuntunse, Ghana, will begin its makeover next month, and the converted dishes will eventually comprise a network of radio telescopes - the African Very Long Baseline Interferometry (VLBI) Network. A VLBI facility can train many geographically distant dishes onto the same astronomical object, and superimposing the signals they collect gives a much higher-resolution image than a solo telescope can obtain. At present, the only member of a VLBI network in Africa is a 26-metre dish at South Africa's radio astronomy hub in Hartebeesthoek, which makes observations with partner telescopes across the world.

The dish in Ghana was donated to the country's science ministry in May by telecommunications company Vodafone Ghana, and after astronomical instruments are fitted it should be operational by June 2013. The African VLBI Network's organizers want to convert three more dishes - in Kenya, Zambia and Madagascar - and build four radio telescopes from scratch in Namibia, Botswana, Mauritius and Mozambique (see 'Dish of the day'). In July, the project received 120 million rand

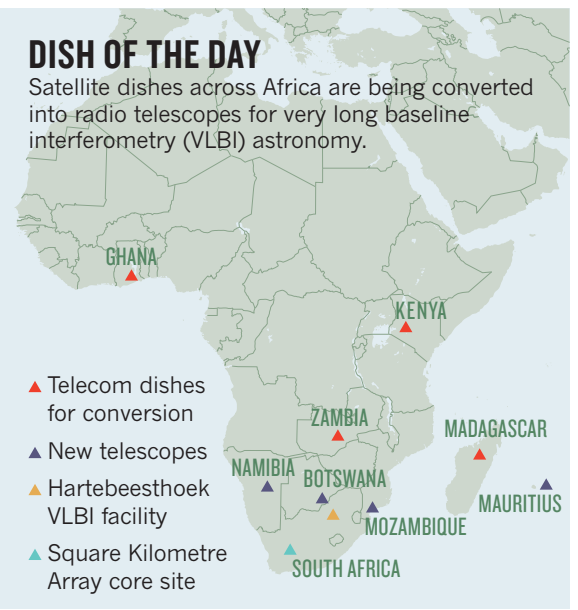

(US\$14.6 million) from the African Renaissance Fund, a development initiative supported by South Africa. A new radio telescope can cost more than 50 million rand, but converting the satellite dishes costs just 15 million to 20 million rand each.

The decades-old equipment can spring some nasty surprises. Last year, an engineer broke the steering mechanism of the dish in Ghana, and its age meant that no spare parts were available, so a completely new control and monitoring system has had to be built.

The project also stands to benefit global VLBI science, says Tom Muxlow of the Jodrell Bank Centre for Astrophysics near Manchester, UK. The most powerful VLBI networks are in the United States and Europe, but equatorial skies are not as well covered - this gap reduces the quality of images available to radio astronomers, potentially missing fine details such as faint jets of plasma from the regions around black holes. "The addition of a dedicated array of African antennas observing the equatorial sky, by itself or in combination with global arrays, has the potential for a truly transformational step in imaging quality," Muxlow says. .

\section{CORRECTION}

The News story 'Republican spending plan casts shadow on science' (Nature 488, 441; 2012) should have said that Paul Ryan's plan could cut spending on R\&D by $5 \%$ below the fiscal-year 2012 budget rather than the President's 2013 request. 